\title{
P161: Knowledge and perception toward hand hygiene among health- care workers in teaching hospital, Korea
}

\author{
EK Kim" ${ }^{*}$, MK Joo ${ }^{1}$, SY Baik', SK Hong ${ }^{2}$ \\ From 2nd International Conference on Prevention and Infection Control (ICPIC 2013) \\ Geneva, Switzerland. 25-28 June 2013
}

\section{Introduction}

Hand hygiene is considered the most important infection control measure in health care setting and forms the core of patient safety. Despite the activity of hand hygiene promotion continued, we observed that hand hygiene compliance is congested. This study was performed in order to survey the knowledge and perception toward hand hygiene for health-care workers(HCWs) to utilize further activity of hand hygiene promotion.

\section{Methods}

This study was performed between January and March 2013 in CHA Bundang medical center, 865-beds teaching hospital in Korea. Our survey material used the WHO questionnaire revised August, 2009. The questionnaire included 7 questions on general characteristics, 10 on knowledge issues( 25 scoring), 11 on perception issues(96 scoring). The collected data were analyzed using the SPSS(ver. 20.0) program.

\section{Results}

During the study period, $348 \mathrm{HCWs}$ were surveyed. The surveyed HCWs were nurses(55.5\%), physicians $(4 \%)$, technicians $(25.6 \%)$, and nurse assistants(14.7\%). The mean age was $30.2(S D=6.6)$ and the majority of participants were female(76.4\%). The mean score of hand hygiene knowledge was $14.25(S D=2.05)$, there were significantly differences in gender (female, $t=-2.276$, $P=.023$ ), clinical experience(above 5years, $t=-2.463$, $P=.014$ ), profession(nurse, $\mathrm{F}=9.337, P<.01$ ) and no significantly differences in age, department. Otherwise the mean score of hand hygiene perception was 75.2
$(S D=11.83)$, there were significantly differences in age (above 31years, $t=-3.224, P=.001$ ), profession(nurse, $\mathrm{F}=4.1, P=.007)$, department(ICU, $\mathrm{F}=2.57, P=.038)$ and no significantly difference in gender, clinical experience. Having had a formal training in hand hygiene was significantly difference both of knowledge $(t=5.50, P<.01)$ and perception $(t=2.4, P=.017)$.

\section{Conclusion}

In this study, the knowledge and perception of hand hygiene for HCWs is low, and It could result in congesting hand hygiene compliance. Therefore, it is necessary to develop a promotion program to build knowledge and perception of hand hygiene to improve compliance for HCWs.

\section{Disclosure of interest}

None declared.

\begin{abstract}
Author details
${ }^{1}$ Infection Control Office, CHA Bundang Medical Center, CHA University, Soengnam-si, Gyeonggi-do, Korea, Republic Of. ${ }^{2}$ Department of internal medicine, CHA Bundang Medical Center, CHA University, Soengnam-si, Gyeonggi-do, Korea, Republic Of.
\end{abstract}

Published: 20 June 2013

doi:10.1186/2047-2994-2-S1-P161

Cite this article as: Kim et al:: P161: Knowledge and perception toward hand hygiene among health- care workers in teaching hospital, Korea. Antimicrobial Resistance and Infection Control 2013 2(Suppl 1):P161. 